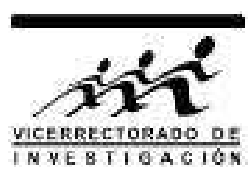

\title{
Restricción del índice espectral usando agujeros negros primordiales
}

\author{
J. Yana Galarza*1, T. Vargas Auccalla ${ }^{1}$, V. D. Vera Cervantes $^{1,2}$ y M. L. Aguilar Hurtado ${ }^{1}$ \\ ${ }^{1}$ Facultad de Ciencias Físicas, Universidad Nacional Mayor de San Marcos, Ap-Postal 14-0149, Lima 14, Perú \\ ${ }^{2}$ Department of Astronomy, University of Bologna, Via Ranzani 1, Bologna, Italy
}

Recibido 30 setiembre 2013 - Aceptado 26 octubre 2013

\begin{abstract}
Analizamos el proceso de colapso gravitacional usado por Press-Schechter para predecir la existencia de los Agujeros Negros Primordiales, PBHs -por sus siglas en inglés-, utilizando la restricción de Josan y los últimos valores de los parámetros cosmológicos obtenidos por el satélite espacial Planck. Asumiendo que las perturbaciones del Universo temprano tuvieron fluctuaciones gaussianas, encontramos que un agujero negro primordial con masa $M>10^{12} \mathrm{~kg}$, aún no debería haberse evaporado. Si comparamos con la fracción de masa, $\beta_{P B H}=10^{-20}$ y asumimos también que la densidad de energía de los PBHs no deben exceder la densidad de energía de la materia oscura fría, CDM -por sus siglas en inglés-, restringimos el índice espectral (blue spectra) a $n_{s}<1.4$ y obtenemos un espectro de potencia de $\mathcal{P}_{\zeta} \approx 10^{-2}$.

Palabras claves: Agujeros negros primordiales, índice espectral, espectro de potencias.
\end{abstract}

\section{Constraint on the spectral index from primordial black holes}

We analyse the process of gravitational collapse using Press-Schechter theory in order to predict the existence of Primordial Black Holes, PBHs, using the Josan's restriction and the latest cosmological parameters measured by Planck satellite. Assuming a gaussian fluctuation on the perturbation in the early Universe, we find that $\mathrm{PBH}$ s with mass $M>10^{12} \mathrm{~kg}$, have not evaporated yet. Comparing with the mass fraction $\beta_{P B H}=10^{-20}$ and assuming that the PBHs energy density should not exceed the Cold Dark Matter, CDM, energy density, we constraint the spectral index (blue espectra) to $n_{s}<1.4$ and obtain a value of the power spectrum $\mathcal{P}_{\zeta} \approx 10^{-2}$.

Keywords: Primordial black hole, spectral index, power spectrum.

Se conjetura que los $\mathrm{PBH}$ se formaron en una época muy temprana en la evolución del Universo, al término de la era inflacionaria cuando comenzaba la época de la radiación. Por tanto, los PBHs serían muy útiles al proporcionar un límite, a escalas muy pequeñas [1-4], en el espectro de potencia de la perturbación primordial de curvatura $\mathcal{P}_{\zeta}$, -que nos brinda información de las fluctuaciones primordiales que son generadas por el campo escalar curvatón $\phi$, que es el responsable de la inflación del Universo-, asimismo, con el que podríamos tener un mejor análisis del escenario de la inflación cuando el Universo estaba apenas en sus primeras etapas de evolución [1, 4, 8 ].

Las observaciones cosmológicas abarcan un rango de escalas como los números de onda comóviles que varían entre $k \sim 1 \mathrm{Mpc}^{-1}$ y $k \sim 10^{-3} \mathrm{Mpc}^{-1}$ [2, 3, 9], donde se prueba una región limitada del potencial de inflación gracias a misiones como el COBE [10], WMAP [11] y el PLANCK [12. Los límites de los $\mathrm{PBH}$ s sobre el espectro de potencia de la curvatura son bastante débiles, el límite superior es de muchos órdenes de magnitud más grandes que las escalas cosmológicas. Sin embargo, podrían aplicarse sobre un amplio rango de escalas que abarca desde $k \sim 10^{-2} \mathrm{Mpc}^{-1}$ hasta $k \sim 10^{23} \mathrm{Mpc}^{-1}$, proporcionando un límite útil sobre los modelos de inflación.

En la época temprana del Universo hubieron colapsos gravitacionales debido a la presencia de inhomogenidades que dieron lugar al Universo que hoy en día conocemos, se cree que muchos de estos colapsos gravitacionales terminaron en los PBHs. Lamentablemente, hasta ahora no se ha podido probar la existencia de estos [11,12], se espera que los futuros datos del satélite PLANCK nos brinde mayor información acerca de la naturaleza de los PBHs.

Un método viable para predecir la existencia de los $\mathrm{PBH}$ es el Blue Spectra, que representa la amplitud de la densidad de las fluctuaciones que debe ser mucho mayor en las pequeñas escalas para la formación de los $\mathrm{PBHs}$ que en las escalas más grandes probadas por la Radiación Cósmica de Fondo, CMB. Esto se consigue aumentando

*ramstojh@gmail.com 
el índice espectral $n_{s}>1$. En este trabajo apostamos por el método del Blue Spectra para calcular el índice espectal y luego restringirlo con los resultados de Josan [13.

En la próxima sección explicamos como se forma un $\mathrm{PBH}$, luego en las dos siguientes introducimos toda la estadística para determinar la abundancia de los $\mathrm{PBH}$ en un escenario gaussiano a una escala de suavización $k \approx 10^{16}$ $\mathrm{Mpc}^{-1}$, esta escala está en el rango de validez, el rango de suavización $R=1 / k \approx 10^{16} \mathrm{Mpc}$ es calculado con nuestro método en la última sección.

\section{La formación de los PBHs}

La fluctuación de la densidad de energía produce que algunas regiones del Universo contengan suficiente energía negativa para eventualmente detener la expansión y colapsar debido a la fuerza de gravedad y luego caer dentro del radio de Schwarzschild. Para los $\mathrm{PBH}$ s siempre podemos considerar fluctuaciones que están inicialmente en una escala más grande que el horizonte. En cosmología moderna se suele referir al horizonte de Hubble como el horizonte, esto es conveniente para que así todas las fluctuaciones de energía puedan ser consideradas solamente como densidad de perturbaciones. Esto se asume para que las perturbaciones de densidad sean las responsables de la formación de los PBHs. Aquí despreciamos las irregularidades tales como la turbulencia y la vorticidad, o alguna otra inhomogeneidad que actúan sólo en escalas más pequeñas que la del horizonte [14].

Para que los $\mathrm{PBH}$ sean formados necesitamos encontrar las condiciones iniciales que estos tuvieron cuando la época de la inflación estaba terminando y comenzada la época de la radiación. Siendo $c_{s}$ la velocidad del sonido, $p$ la presión y $\rho$ la densidad, afirmamos que

$$
\begin{aligned}
c_{s} & =\frac{c}{\sqrt{3}}, \\
p & =c_{s}^{2} \rho=\frac{\rho}{3},
\end{aligned}
$$

donde $c$ es la velocidad de luz.

Para que una parte del Universo colapse y se forme un $\mathrm{PBH}$, la métrica de los $\mathrm{PBH}$ debe ser la métrica de Schwarzschild gobernado por la curvatura positiva $K>1$ en la ecuación de Friedman. Así podemos considerar una región de simetría esférica con densidad $\tilde{\rho}$ más grande que la densidad de fondo $\rho$.

La ecuación de Friedman para la métrica de curvatura positiva esta dada por,

$$
\tilde{H}^{2}(\tilde{t})=\frac{8 \pi}{3 M_{p l}^{2}} \tilde{\rho}(\tilde{t})-\frac{1}{\tilde{a}^{2}(\tilde{t})},
$$

donde $M_{p l}$ es la masa de Planck, $\tilde{a}(\tilde{t})$ es el factor de escala y $\tilde{H}(\tilde{t})$ es el parámetro de Hubble. El espacio de fondo con curvatura plana es

$$
H^{2}=\frac{8 \pi}{3 M_{p l}^{2}} \rho(t) .
$$

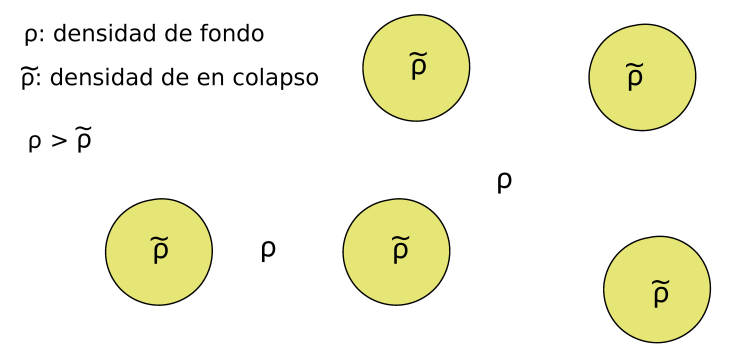

Figura 1: Región del Universo con inhomogeneidades de densidad donde $\rho$ es la densidad de fondo del universo no perturbado con curvatura $K=0$, y $\tilde{\rho}$ es la densidad de los cuerpos colapsando gravitacionalmente con curvatura $K>1$ debido a que han alcanzado el límite de la perturbación de la densidad crítica $\delta_{c}=1 / 3$.

Consideramos las coordenadas iniciales $t=\tilde{t}=t_{i}$ y $\tilde{H}=H=H_{i}$, teniendo en cuenta que al inicio la distribución de las densidades era homogénea. Además usando la Ec. (3) y la Ec. (4) encontramos,

$$
\delta_{i} \equiv \frac{\tilde{\rho}_{i}-\rho_{i}}{\rho_{i}}=\frac{1}{H_{i}^{2} R_{i}^{2}} .
$$

Esta ecuación es conocida como la perturbación de densidad inicial [15], y se cumple para la condición inicial cuando la perturbación cruza el horizonte,

$$
\frac{1}{3} \lesssim \delta_{c} \lesssim 1
$$

Esta es la principal condición para que los $\mathrm{PBH}$ se formen con una masa aproximadamente igual a la masa del horizonte [16], utilizando el valor crítico $\delta_{c}=1 / 3$ para la formación de los PBHs, Fig. 1 .

\section{Cálculo estándar}

La varianza de la masa es el valor medio cuadrático de la sobredensidad $\tilde{\rho}$ en una región dada del espacio, que es expresada en términos del espectro de densidad en el super horizonte o la fluctuación de la curvatura dada por la inflación, ver apéndice A Asumiendo una estadística gaussiana para la densidad de fluctuaciones, la probabilidad para encontrar el promedio de la densidad de contraste $\delta$ en una región esférica de tamaño $R$ esta dada por

$$
P(\delta(R))=\frac{1}{\sqrt{2 \pi} \sigma_{\delta}(R)} \exp \left(-\frac{\delta^{2}(\mathrm{R})}{2 \sigma_{\delta}^{2}(\mathrm{R})}\right),
$$

donde $\sigma_{\delta}(R)$ es la varianza de masa suavizada a una escala $R$ siendo calculada por la convolución del espectro de potencias con una función de ventana, considerando que el campo de fluctuaciones en la densidad, $\delta(\mathbf{x})$ no es una 
función suave con respecto a la posición $x$, sino se suaviza convolucinándolo con una función ventana (window function) dada por

$$
\sigma_{\delta}^{2}(R)=\int_{0}^{\infty} W^{2}(k R) \mathcal{P}_{\delta}(k) \frac{d k}{k} .
$$

En la expresión anterior, $P_{\delta}(k)$ es definida por

$$
\left\langle\delta_{k} \delta_{k^{\prime}}^{*}\right\rangle \equiv \frac{2 \pi^{2}}{k^{3}} \mathcal{P}_{\delta}(k) \delta\left(k-k^{\prime}\right),
$$

y la función ventana que usaremos es una gaussiana que está dado por,

$$
W(x)=\exp \left[-\frac{x^{2}}{2}\right],
$$

donde $x \equiv k R$. Existen otros tipos de función ventana pero para nuestro cálculo optamos por el gaussiano, ver apéndice $B$. En las hipersuperficies comóviles hay una simple relación entre la densidad de la perturbación y la perturbación de la curvatura [17],

$$
\delta(t, k)=\frac{2(1+w)}{5+3 w}\left(\frac{k}{a H}\right)^{2} \zeta(k),
$$

donde $\zeta$ es la perturbación de la curvatura y $w$ es el parámetro de estado. El espectro de potencia esta relacionada por

$$
\mathcal{P}_{\delta}(k, t)=\frac{4(1+w)^{2}}{(5+3 w)^{2}}\left(\frac{k}{a H}\right)^{4} \mathcal{P}_{\zeta}(k) .
$$

Luego cuando se cruza el horizonte, ver Fig. 2 $k=a H$, obtenemos,

$$
\mathcal{P}_{\delta}(k, t)=\frac{4(1+w)^{2}}{(5+3 w)^{2}} \mathcal{P}_{\zeta}(k)
$$

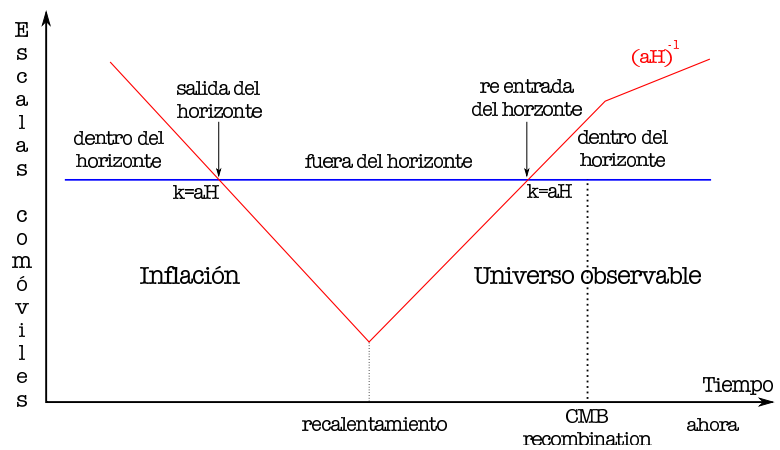

Figura 2: Escala comóvil de Hubble como una función del factor escalar. Observamos que el universo se separa en dos partes una en la época inflacionaría (lado izquierdo) y después de la reentrada del horizonte un Universo observable a partir del CMB (lado derecho). La entrada y salida del horizonte esdeterminan las perturbaciones primordiales.
Para calcular la fracción del universo el cuál excede el límite para la formación de los $\mathrm{PBH}$ es dado por la teoría de Press-Schechter [18],

$$
\beta_{P B H}=2 \int_{\delta_{c}}^{\infty} P(\delta(M)) d \delta(M) .
$$

En el presente trabajo asumimos que el espectro primordial de potencia está dado por

$$
\mathcal{P}_{\zeta}(k)=A_{\zeta}\left(\frac{k}{k_{0}}\right)^{n_{s}-1}
$$

donde $A_{\zeta}=\left(2.196_{-0.060}^{+0.051}\right) \times 10^{-9}$ con un índice espectral $n_{s}=0.9603 \pm 0.0073$ con un punto de pivote $k_{0}=0.002$ $\mathrm{Mpc}^{-1}$, según los últimos datos obtenidos por Planck [12].

\section{La abundancia de los PBHs}

La restricción de la abundancia de los $\mathrm{PBH}$ puede ser encontrado de dos formas, aquellos que surgen de las consecuencias gravitacionales en la época presente [18] y aquellos que surgen del producto de su evaporación [19]. Con el fin de restringir la densidad primordial de la perturbación de curvatura, necesitamos trasladar las restricciones dentro de los límites de la fracción de la masa inicial de los PBHs.

Asumimos que el horizonte de la masa de los $\mathrm{PBH}$ es $M_{H}=3^{3 / 2} M_{P B H}$ [13]. Tomando en cuenta la expansión cosmológica, la fracción de la masa inicial de los PBHs, $\beta_{P B H}$, está relacionada con la densidad presente de los PBHs, $\Omega_{P B H}$ [13], por

$$
\beta_{P B H}=6.4 \times 10^{-19} \Omega_{P B H}\left(\frac{M_{P B H}}{3^{3 / 2} 5 \times 10^{14} \mathrm{~g}}\right)^{1 / 2} .
$$

Como fue mostrado por Hawking [19], un agujero negro con masa $M_{P B H}$ emite radiación térmica con temperatura,

$$
T_{B H} \approx 1.06\left(\frac{10^{13} \mathrm{~g}}{M_{P B H}}\right) \mathrm{GeV},
$$

donde el tiempo de vida de un PBH está dado por [20]

$$
\tau \approx 6.24 \times 10^{-27} M_{P B H}^{3} f\left(M_{P B H}\right)^{-1} \mathrm{~s},
$$

y para las masas ligeras de $\mathrm{PBHs} f\left(M_{P B H}\right)=1.569$. Del último resultado del satélite Planck [12], la presente era del universo es $13.82 \mathrm{Gyr}$ y la masa inicial de un $\mathrm{PBH}$ el cual se está evaporando hoy en día es $M_{P B H}=$ $4.76 \times 10^{14} \approx 5 \times 10^{14} \mathrm{~g}$, mientras los menos masivos ya se han evaporado en la época actual.

Estamos interesados en los PBHs que se están evaporando en la actualidad y por tanto usaremos restricciones gravitacionales. Para esto debemos calcular la densidad hoy en día de los PBHs con $M_{P B H}>5 \times 10^{14}$, los cuales aún no se han evaporado, y estos deberían ser menores que el límite superior de la densidad de la materia oscura fria (CDM) que se mide hoy en día [13, 21], 
ver apéndice C. Usando los datos del satélite Planck [12], $\Omega_{\mathrm{CDM}} h^{2}=0.1199 \pm 0.0027, h=0.719$ obtenemos

$$
\Omega_{P B H}<0.23
$$

el cual usando la Ec. [16], obtenemos

$$
\beta_{P B H}<1.4 \times 10^{-19}\left(\frac{M_{P B H}}{3^{3 / 2} 5 \times 10^{14} \mathrm{~g}}\right)^{1 / 2},
$$

para una masa $M_{P B H}>5 \times 10^{14} \mathrm{~g}$. Para esta masa, la fracción de masa de los $\mathrm{PBH}$ s no debe de exceder $\beta_{P B H} \approx 10^{-20}$. A partir de este resultado restringimos los límites del espectro de potencia de la perturbación de la curvatura y con ello también el índice espectral.

\section{Nuestro Cálculo}

Para un espectro de fluctuación gaussiana, la fracción de densidad actual de los $\mathrm{PBH}$ s han sido típicamente usados para restringir el índice espectral bajo las asunciones de un espectro de escala libre, scale-free spectrum [1,2 14 22]. El límite resultante, $n_{s} \lesssim 1.3$, basado por ejemplo en VERITAS buscado en $k=3 \times 10^{15} \mathrm{Mpc}^{-1}$, [23], es fácilmente satisfecha por el reciente valor medido $n_{s}=0.968 \pm 0.0012$ [24]. En términos de generalización, la escala invariante gaussiana del poder espectral de los $\mathrm{PBH}$ s han sido usados para límitar la amplitud de la perturbación de curvatura en un nivel de $\mathcal{P}_{R} \lesssim 10^{-2}$ [3, 25], en escalas correspondientes a $10^{-2}<k<10^{19} \mathrm{Mpc}^{-1}$.

Los límites observacionales recientes de los $\mathrm{PBHs}$ [3, 26], limita $\beta_{P B H}$ con valores en un rango de $\beta_{P B H} \lesssim$ $10^{-10}$ para masas solares de PBHs, hasta $\beta_{P B H} \lesssim 10^{-28}$ para un pequeño rango de masas de $\mathrm{PBH}$, y alrededor de $10^{13}$ g y $\beta_{P B H} \lesssim 10^{-20}$ para masas más pequeñas.

Para usar nuestros resultados, necesitamos relacionar la escala comóvil de suavización $R$ con el horizonte de masa. La masa de los PBHs $M_{P B H}$ que corresponde al horizonte de masa es $M_{H}=3^{3 / 2} M_{P B H}$ [3], y su respectivo número de onda, ver apéndice [D], está dado por

$\frac{k}{k_{e q}}=\left(2 \times 3^{3}\right)^{-1 / 4}\left(\frac{g_{*}^{H}}{g_{*}^{e q}}\right)^{1 / 4}\left(\frac{g_{S}^{e q}}{g_{S}^{R}}\right)^{1 / 3}\left(\frac{M_{e q}}{M_{P B H}}\right)^{1 / 2}$,

donde $k_{e q}=0.072 \Omega_{m} h^{2} \mathrm{Mpc}^{-1}=9.68 \times 10^{-3} \mathrm{Mpc}^{-1}$ [24] y $M_{e q}=3.5 \times 10^{17} M_{\odot}$ [27] son los números de onda y el horizonte de masa, respectivamente igualados, y $g_{S}^{e q}=3.91, g_{*}^{e q}=3.36, g_{*}^{H} \simeq g_{S}^{H} \geqslant 106.75$ [28], son los respectivos grados de libertad relativistas igualados en el tiempo de ingreso al horizonte.

Por definición, la región suavizada obedece $R=1 / k$, y de la Ec. (21) podemos encontrar que $R \simeq 10^{-16} \mathrm{Mpc}$ que es el rango de interes donde se forman los PBHs. Es característica de la formación de objetos masivos como los $\mathrm{PBH}$ formarse en la cola de distribución de probabilidad, Fig. (3), a medida que aumentamos el índice espectral
Blueshift, $n_{s}$, es más probable la formación de los $\mathrm{PBHs}$, con este método limitamos el índice espectral con la restricción que hicimos anteriormente.

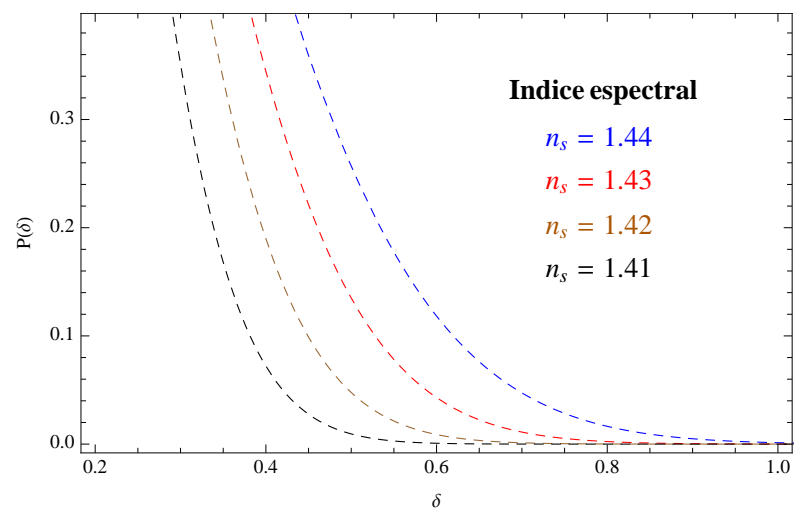

Figura 3: Con el aumento del índice espectral $n_{s}$, la probabilidad de formación de los $\mathrm{PBH}$ es mayor, la figura muestra un índice espectral desde un valor de 1.41 hasta 1.44 .

Usando la Ec. (15) y comparandola con la Ec. (20) obtenemos un índice espectral $n_{s}=1.33$, (Fig. (4) con un espectro de potencia $\mathcal{P}_{\zeta} \approx 10^{-2}$.

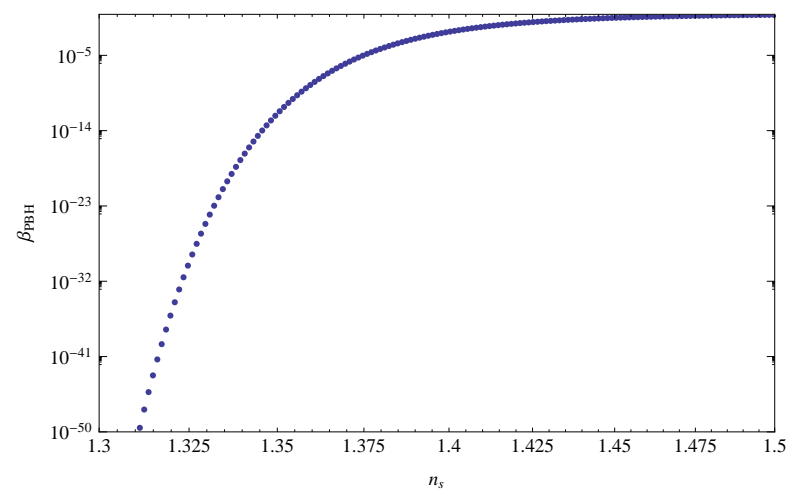

Figura 4: Nuestro resultado muestra que para un $\beta_{P B H}=$ $10^{-20}$ le corresponde un índice espectral $n_{s}=1,33$.

\section{Conclusiones}

Los PBHs son una fuente de limitación del espectro de potencia en escalas muy pequeñas, debido a que pudieron haberse formado en el Universo en la época de la radiación. Hemos desarrollado un nuevo método para calcular el rango de suavización de los $\mathrm{PBHs}, R=1 / k$, utilizando los datos obtenidos por el satétile Planck, el cual nos dio una escala aproximada de $k=10^{16} \mathrm{Mpc}$, el cual está de acuerdo a las escalas en las que pudieron haberse formado los PBHs.

Nuestro cáculo nos muestra una restricción al índice espectral $n_{s}<1.4$, con un espectro de potencia 
$\mathcal{P} \approx 10^{-2}$, para una masa $M_{P B H}=10^{13} \mathrm{~kg}$ y una fracción de masa $\beta_{P B H}=10^{-20}$, los cuales aún están en proceso de evaporación en la actualidad.

\section{Agradecimientos}

Este artículo tiene sus inicios en el Perimeter Institute for Theoretical Physics, en colaboración con Sarah Shandera a quién se le agradece sus aportes, sugerencias e incentivo en la elaboración del presente trabajo.

\section{Apéndices}

\section{A. Cálculo de la Varianza en el espectro de perturbación}

Asumamos que el universo está dividido en muchas regiones de volumen $V_{u}$, en los cuales existen perturbaciones que se definen como una superposición de ondas planas que evolucionan de manera independiente, mientras que las fluctuaciones permanecen lineales. Luego representamos la distribución como componentes independientes en un espacio de las transformadas de Fourier, en términos de los vectores de onda de cada componente $\boldsymbol{k}$.

Consideramos la densidad media $\langle\rho\rangle$ en un volumen $V_{u}$ y $\rho(\boldsymbol{x})$ la densidad en un punto especificado por el vector posición $\boldsymbol{x}$ con respecto a algún origen arbitrario. Usualmente definimos las fluctuaciones $\delta(\boldsymbol{x})=$ $(\rho(\boldsymbol{x})-\langle\rho\rangle) /\langle\rho\rangle$. Expresando como una serie de Fourier,

$$
\delta(\boldsymbol{x})=\sum_{\boldsymbol{k}} \delta_{\boldsymbol{k}} \exp [i \boldsymbol{k} \cdot \boldsymbol{x}],
$$

donde $\delta_{k}$ son las cantidades complejas dadas, esto es muy sencillo de ver por

$$
\delta_{\boldsymbol{k}}=\frac{1}{V_{u}} \int_{V_{u}} \delta(\boldsymbol{x}) \exp (-i \boldsymbol{k} \cdot \boldsymbol{x}) d \boldsymbol{x} .
$$

Su varianza esta dada por

$$
\sigma^{2} \equiv\left\langle\delta^{2}\right\rangle=\sum_{k}\left\langle\left|\delta_{k}\right|^{2}\right\rangle=\frac{1}{V_{u}} \sum_{k} \delta_{k}^{2}
$$

donde el promedio es tomado sobre todos los ensembles de realización. $\left\langle\left|\delta_{k}\right|^{2}\right\rangle$ es la contribución de la varianza debido a los números de onda $\boldsymbol{k}$. Si ahora tomamos el límite $V_{u} \rightarrow \infty$ y asumimos que el campo de densidad es estadísticamente homogéneo e isotrópico, así que no hay una dependencia en la dirección de $\boldsymbol{k}$ pero solo sobre $k=|\boldsymbol{k}|$, podemos escribir la Ec. (24) de la siguiente forma

$$
\sigma^{2}=\frac{1}{V_{u}} \sum_{\mathbf{k}} \delta_{k}^{2} \rightarrow \frac{1}{2 \pi^{2}} \int_{0}^{\infty} \mathcal{P}(k) k^{2} d k,
$$

donde $\delta_{k}^{2}=\mathcal{P}(k)$ en el límite $V_{u} \rightarrow \infty$. La cantidad $\mathcal{P}(k)$ es llamada el poder espectral de la función de densidad del campo $\delta$ o power spectrum. La varianza no depende de la posición espacial pero sí del tiempo, porque las amplitudes de la perturbación $\delta_{k}$ evolucionan. La cantidad $\sigma^{2}$ nos da información acerca de las amplitudes de las perturbaciones.

La Ec. (25) también puede ser escrito en la forma

$$
\sigma^{2}=\frac{1}{2 \pi^{2}} \int_{0}^{\text {inf }} \mathcal{P}(k) k^{2} d k=\int_{-\infty}^{+\infty} \Delta(k) d \ln k,
$$

donde la cantidad adimensional es dada por

$$
\Delta(k)=\frac{1}{2 \pi^{2}} \mathcal{P}(k) k^{3}
$$

que representa la contribución de la varianza por unidad logaritmica en el intervalo de $\boldsymbol{k}$. La Ec. (26) claramente no converge y es necesario filtrar el spectrum para conseguir resultado válidos.

\section{B. Cálculo de la varianza de la masa y el filtro función ventana}

La Ec. (26) no converge y sus resultados podrían ser infinitos, por tanto es coveniente construir una descripción estadística del campo de fluctuaciones como una función de alguna escala de "suavización" $R$. Sea $\langle M\rangle$ la masa media dentro de un volumen esférico $V$ y radio $R$ :

$$
\langle M\rangle=\langle\rho\rangle V=\frac{3}{4} \pi\langle\rho\rangle R^{3} .
$$

Luego la varianza de las masas dentro del volumen $V$ está dado por

$$
\sigma_{\delta}^{2}(R)=\frac{\left\langle(M-\langle M\rangle)^{2}\right\rangle}{\langle M\rangle^{2}}=\frac{\left\langle\delta M^{2}\right\rangle}{\langle M\rangle^{2}},
$$

donde el promedio es realizado sobre todos los volumenes $V ; \sigma_{\delta}(R)$ es la raíz cuadrada media de la fluctuación de la masa. Usando la Ec. (22) y Ec. (29) se convierte,

$$
\begin{aligned}
\sigma_{\delta}^{2}(R)=\frac{1}{V^{2}}\left\langle\int_{V} \int_{V} \sum_{\boldsymbol{k}} \delta_{k} \exp [i \boldsymbol{k} \cdot \boldsymbol{x}]\right. \\
\left.\quad \times \sum_{\boldsymbol{k}^{\prime}} \delta_{k^{\prime}} \exp \left[i \boldsymbol{k}^{\prime} \cdot \boldsymbol{x}^{\prime}\right] d \boldsymbol{x} d \boldsymbol{x}^{\prime}\right\rangle
\end{aligned}
$$

y operando las integrales obtenemos,

$$
\sigma_{\delta}^{2}(R)=\frac{1}{V^{2}}\left\langle\sum_{\boldsymbol{k}, \boldsymbol{k}^{\prime}} \delta_{\boldsymbol{k}} \delta_{\boldsymbol{k}}^{\prime *} \exp \left[i\left(\boldsymbol{k}-\boldsymbol{k}^{\prime}\right) \cdot \boldsymbol{x}_{0}\right] I_{1} I_{2}\right\rangle,
$$

donde

$$
I_{1}=\int_{V} \exp \left[i \boldsymbol{k} \cdot\left(\boldsymbol{x}-\boldsymbol{x}_{0}\right)\right] d\left(\boldsymbol{x}-\boldsymbol{x}_{0}\right)
$$

y

$$
I_{2}=\int_{V} \exp \left[-i \boldsymbol{k}^{\prime} \cdot\left(\boldsymbol{x}^{\prime}-\boldsymbol{x}_{0}\right)\right] d\left(\boldsymbol{x}^{\prime}-\boldsymbol{x}_{0}\right)
$$


Entonces resulta

$$
\begin{array}{r}
\sigma_{\delta}^{2}(R)=\sum_{\boldsymbol{k}}\left\langle\left|\delta_{k}\right|^{2}\right\rangle\left[\frac{1}{V} \int_{V} \exp [i \boldsymbol{k} \cdot \boldsymbol{y}] d \boldsymbol{y}\right]^{2}= \\
\frac{1}{V_{u}} \sum_{\boldsymbol{k}} \delta_{k}^{2} W^{2}(k R) .
\end{array}
$$

En las ecuaciones anteriores $\boldsymbol{x}_{0}$ es el centro de una esfera de volumen $V$, y la media es tomada sobre todas las esferas, es decir sobre todos los puntos $\boldsymbol{x}_{0}$. La función $W(K R)$ en la Ec. (34) es llamada "función ventana". Esta función puede ser construida haciendo uso de los armónico esféricos desarrollando $\exp [i \boldsymbol{k} \cdot \boldsymbol{y})$,

$$
\exp [i \boldsymbol{k} \cdot \boldsymbol{y}]=\sum_{l, m} j_{l}(k r) i^{l}(2 l+1) P_{l}^{|m|}(\cos \theta) \exp [i m \varphi],
$$

donde $j_{l}$ son las funciones de Bessel, $P_{l}^{|m|}$ son los polinomios asociados de Legendre, y $r, \theta, \varphi$ son las coordenadas polares esféricas. La integral $I$ de las Ecs. (32 y 33) en la Ec. (34) se convierte en

$$
\begin{aligned}
I=\sum_{l, m} & i^{l}(2 l+1) \int_{0}^{2 \pi} \exp [i m \varphi] d \varphi \\
& \times \int_{0}^{\pi} P_{l}^{|m|}(\cos \theta) \sin \theta d \theta \int_{0}^{R} j_{l}(k r) r^{2} d r
\end{aligned}
$$

○ alternativamente,

$$
I=4 \pi \int_{0}^{R} j_{0}(k r) r^{2} d r=\frac{4 \pi}{k^{3}}(\sin k R-\cos k R)
$$

donde la integral sobre $\theta$ y $\varphi$ son cero bajo $m=l=0$, de esta forma obtenemos la función ventana dada por

$$
W(K R)=\frac{3(\sin k R-k R \cos k R)}{(k R)^{3}},
$$

su comportamiento es tal que $W(x) \simeq 1$ para $x \leq 1 \mathrm{y}$ $|W(x)| \leq x^{-2}$ para $x \gg 1$. Expresando a una distribución contínua de ondas planas, es decir el límite expresado por la Ec. (35), la varianza de masa resulta

$$
\sigma_{\delta}^{2}(R)=\frac{1}{2 \pi^{2}} \int_{0}^{\infty} \mathcal{P}(k) W^{2}(k R) k^{2} d k<\sigma^{2},
$$

el cual, como debería ser, es una función de $\mathrm{R}$ y por tanto de M. El significado de "Función Ventana" es la siguiente: la contribución dominante de $\sigma_{\delta}^{2}(R)$ es desde los componentes de la perturbación con longitudes de onda $\lambda \simeq k^{-1}>R$ porque estas con alta frecuencia tienden a ser promediados dentro del volumen en la función ventana, hemos supuesto tácitamente que el espectro cae cuando decrece $k$, así ondas con grandes longitutes $\lambda$ contribuyen solo en pequeñas cantidades.
También se suele utilizar para el caso gaussiano,

$$
W(K R)=\exp \left[-\frac{k^{2} R^{2}}{2}\right] .
$$

Ambos filtros mencionados se muestran en la Fig. (5).

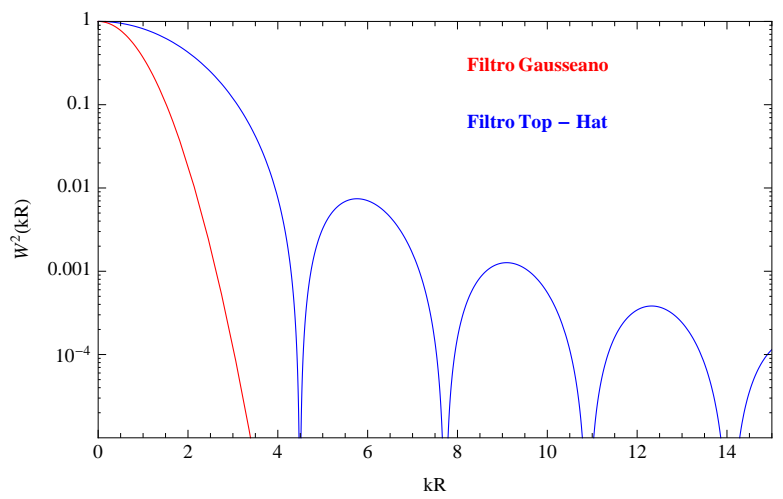

Figura 5: La figura muestra la diferencia entre el filtro Top-hat y el Gaussiano representados en el espacio de Fourier [17]).

\section{Agujeros negros primordiales como ma- teria oscura fría}

Hawking en 1971 mostró que la densidad de fluctuación en un universo temprano podría llevar a colapsos gravitacionales [19], luego Carr y Hawking en 1974 argumentaron que este colapso podría llevar a la formación de agujeros negros alrededor del horizonte de masa $M_{h}$ en un tiempo t [9], donde

$$
M_{h}(t) \approx \frac{c^{3} t}{G} .
$$

Para la ecuación de estado donde la presión $p$ y densidad de energía $\rho$ son dados por $p=\rho / 3$, regiones con sobredensidad $\delta \rho / p \gtrsim 1 / 3$ deberían de colapsar en agujeros negros con masa del orden del horizonte de masa $M_{h}$. Para una ecuación de estado suavizada donde $p \approx 0$, los agujeros negros deberían formarse mucho más facilmente desde pequeñas reliquias de perturbación adiabática.

Durante la transición de fase en un universo temprano, y en particular durante la transición quark-hadrón descrita por la Cromodinámica Cuántica, QCD -por sus siglas en inglés-, en una época cósmica de $\sim 10^{-5}$ segundos, en esta época la teoría predice que la ecuación de estado debe ser suavizado.

Por esta razón Jedamzik en 1997 concluyó que la función de masa de los $\mathrm{PBH}$ deberían de mostrar un pico pronunciado en la escala de masa de la QCD, $M_{Q C D} \approx M_{\odot}$. Estos resultados fueron confirmados por las simulaciones numéricas de hidrodinámica general relativista, el cual muestra que el límite de la densidad de fluctuación $\delta \rho / \rho$ 
para la formación de un agujero negro cae cuando el universo sufre una transición de fase, para producir un espectro de masa dominado por el horizonte de masa en la época de transición.

Yokoyama en 1997 supuso el caso de modelos inflacionarios con múltiples campos escalares, y con una correcta elección de parámetros, produce los $\mathrm{PBH}$ con picos de masa en la escala de masa de los MACHOs. Por tanto es factible pensar que los $\mathrm{PBH}$ s con determinada distribución de masa pueden ser producidos con suficiente abundancia para producir la matería oscura, y con valores naturales para el modelo de parámetros produce masas en el rango de los MACHOs.

\section{Escala comóvil de suavización}

Para trabajar con los PBHs, primero debemos de analizar la relación existente entre la masa del horizonte $M_{H}$ y la masa $M_{e q}$,

$$
\frac{M_{H}}{M_{e q}}=\frac{\rho_{R, H} H_{H}^{-3}}{\rho_{e q} H_{e q}^{-3}}=\frac{H_{e q}}{H_{H}}
$$

de donde,

$$
\begin{aligned}
\frac{H_{e q}}{H_{H}} & =\sqrt{\frac{2 \rho_{R, e q}}{\rho_{R, H}}} \\
& =\sqrt{\frac{2 g_{*}^{e q}}{g_{*}^{H}}} \frac{T_{e q}^{2}}{T_{H}^{2}} \\
& =\sqrt{\frac{2 g_{*}^{e q}}{g_{*}^{H}}} \frac{g_{S H}^{1 / 3} a_{H}}{g_{S e q}^{1 / 3} a_{e q}} \\
& =\sqrt{2}\left(\frac{g_{*}^{e q}}{g_{*}^{H}}\right)^{1 / 2}\left(\frac{g_{S}^{H}}{g_{S}^{e q}}\right)^{2 / 3}\left(\frac{a_{H}}{a_{e q}}\right)^{2}
\end{aligned}
$$

Comparando la Ec. (42) y la Ec. (46) se obtienen

$$
\frac{M_{H}}{M_{e q}}=\frac{H_{e q}}{H_{H}}
$$

y

$$
\frac{a_{H}}{a_{e q}}=\left(\frac{M_{H}}{M_{e q}}\right)^{1 / 2}\left(\frac{g_{*}^{H}}{g_{*}^{e q}}\right)^{1 / 4}\left(\frac{g_{S}^{e q}}{g_{S}^{H}}\right)^{1 / 3} \frac{1}{2^{1 / 4}} .
$$

La relación de números de onda está dado por

$$
\frac{k_{H}}{k_{e q}}=\frac{a_{H} H_{H}}{a_{e q} H_{e q}}
$$

y combinando la Ec. (46) en la Ec. (49) obtenemos,

$$
\begin{gathered}
\frac{k_{H}}{k_{e q}}=\left(\frac{a_{H}}{a_{e q}}\right) \frac{1}{\sqrt{2}}\left(\frac{g_{*}^{H}}{g_{*}^{e q}}\right)^{\frac{1}{2}}\left(\frac{g_{S}^{e q}}{g_{S}^{H}}\right)^{\frac{2}{3}}\left(\frac{a_{e q}}{a_{H}}\right)^{2}=\frac{2^{\frac{1}{4}}}{\sqrt{2}} \\
\left(\frac{g_{*}^{H}}{g_{*}^{e q}}\right)^{\frac{1}{2}}\left(\frac{g_{S}^{e q}}{g_{S}^{H}}\right)^{\frac{2}{3}}\left(\frac{M_{e q}}{M_{H}}\right)^{\frac{1}{2}}\left(\frac{g_{*}^{e q}}{g_{*}^{M}}\right)^{\frac{1}{4}}\left(\frac{g_{S}^{H}}{g_{S}^{e q}}\right)^{\frac{1}{3}} \cdot(50)
\end{gathered}
$$

Introduciendo la Ec. [48] en la Ec. (50), obtenemos

$$
\frac{k_{H}}{k_{e q}}=\frac{1}{2^{1 / 4}}\left(\frac{g_{*}^{H}}{g_{*}^{e q}}\right)^{\frac{1}{4}}\left(\frac{g_{S}^{e q}}{g_{S}^{H}}\right)^{\frac{1}{3}}\left(\frac{M_{e q}}{M_{H}}\right)^{\frac{1}{2}} .
$$

La masa de los PBHs $M_{P B H}$ que corresponde al horizonte de masa es $M_{H}=3^{3 / 2} M_{P B H}$, introduciendo esto en la Ec. (51) obtenemos finalmente

$$
\begin{aligned}
\frac{k}{k_{e q}}=\frac{1}{\left(2 \times 3^{3}\right)^{\frac{1}{4}}}\left(\frac{g_{*}^{R}}{g_{*}^{e q}}\right)^{\frac{1}{4}} \times & \\
& \left(\frac{g_{S}^{e q}}{g_{S}^{R}}\right)^{\frac{1}{3}}\left(\frac{M_{e q}}{M_{P B H}}\right)^{\frac{1}{2}} .
\end{aligned}
$$

\section{Referencias}

[1] A. M. Green y A. R. Liddle, Phys. Rev. D 56, 6166 (1997), astro-ph/9704251.

[2] A. M. Green, A. R. Liddle, K. A. Malik, y M. Sasaki, Phys. Rev. D 70, 041502 (2004), astro-ph/0403181

[3] A. S. Josan, A. M. Green, y K. A. Malik, Phys. Rev. D 79, 103520 (2009a), 0903.3184

[4] H. V. Peiris y R. Easther, J. Cosmol. Astrop. Phys. 0807, 024 (2008), 0805.2154.

[5] B. J. Carr, J. Gilbert, y J. E. Lidsey, Phys. Rev. D 50, 4853 (1994), astro-ph/9405027

[6] T. Bringmann, C. Kiefer, y D. Polarski, Phys. Rev. D 65, 024008 (2002), astro-ph/0109404
[7] S. M. Leach, I. J. Grivell, y A. R. Liddle, Phys. Rev. D 62, 043516 (2000), astro-ph/0004296.

[8] K. Kohri, D. H. Lyth, y A. Melchiorri, J. Cosmol. Astrop. Phys. 0804, 038 (2008), 0711.5006.

[9] B. J. Carr y S. W. Hawking, Monthly Notices of the Royal Astronomical Society 168, 399 (1974).

[10] K. Górski, A. Banday, C. Bennett, G. Hinshaw, A. Kogut, G. Smoot, y E. Wright, The Astrophysical Journal Letters 464, L11 (1996).

[11] E. Komatsu, K. Smith, J. Dunkley, C. Bennett, B. Gold, G. Hinshaw, N. Jarosik, D. Larson, M. Nolta, L. Page, et al., The Astrophysical Journal Supplement Series 192, 18 (2011a). 
[12] P. Collaboration, P. Ade, N. Aghanim, C. ArmitageCaplan, M. Arnaud, M. Ashdown, F. Atrio-Barandela, J. Aumont, C. Baccigalupi, A. Banday, et al., arXiv preprint arXiv:1303.5076 (2013).

[13] A. S. Josan, A. M. Green, y K. A. Malik, Phys. Rev. D 79, 103520 (2009).

[14] J. S. Bullock y J. R. Primack, Phys. Rev. D 55, 7423 (1997), astro-ph/9611106

[15] J. C. Niemeyer y K. Jedamzik, Phys. Rev. Lett. 80, 5481 (1998).

[16] D. Nadezhin, I. Novikov, y A. Polnarev, Soviet Astronomy 22, 129 (1978).

[17] A. R. Liddle y D. H. Lyth, Cosmological inflation and large-scale structure, Cambridge University Press, Cambridge (2000).

[18] W. H. Press y P. Schechter, The Astrophysical Journal 187, 425 (1974).

[19] S. W. Hawking, Nature 248, 30 (1974).
[20] J. H. MacGibbon y B. J. Carr, The Astrophysical Journal 371, 447 (1991).

[21] M. Hawkins, Monthly Notices of the Royal Astronomical Society 415, 2744 (2011).

[22] S. Chongchitnan y G. Efstathiou, J. Cosmol. Aastrop. Phys. 0701, 011 (2007), astro-ph/0611818

[23] G. Tešić et al, J. Physics: Conference Series 375, 052024 (2012).

[24] E. Komatsu et al. (WMAP Collaboration), Astrophys. J. Suppl. 192, 18 (2011b), 1001.4538

[25] L. Alabidi, K. Kohri, M. Sasaki, y Y. Sendouda, J. Cosmol. Astrop. Phys. 1209, 017 (2012), 1203.4663.

[26] B. Carr, K. Kohri, Y. Sendouda, y J. Yokoyama, Phys. Rev. D 81, 104019 (2010), 0912.5297.

[27] P. Scott y S. Sivertsson, Phys. Rev. Lett. 103, 211301 (2009), 0908.4082.

[28] E. Kolb y M. Turner, The Early Universe, Frontiers in Physics Vol. 69, Addison-Wesley, New York (1990). 